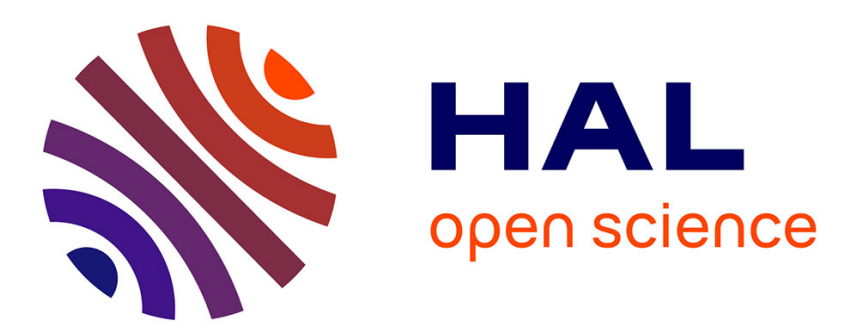

\title{
Assessment of ZDES for WMLES of turbulent boundary layers with pressure gradient and mild flow separation
}

\author{
Jaime Vaquero, Nicolas Renard, Sébastien Deck
}

\section{To cite this version:}

Jaime Vaquero, Nicolas Renard, Sébastien Deck. Assessment of ZDES for WMLES of turbulent boundary layers with pressure gradient and mild flow separation. AIAA AVIATION 2021 FORUM, Aug 2021, VIRTUAL EVENT, United States. 10.2514/6.2021-2811 . hal-03386097

\section{HAL Id: hal-03386097 https://hal.science/hal-03386097}

Submitted on 19 Oct 2021

HAL is a multi-disciplinary open access archive for the deposit and dissemination of scientific research documents, whether they are published or not. The documents may come from teaching and research institutions in France or abroad, or from public or private research centers.
L'archive ouverte pluridisciplinaire HAL, est destinée au dépôt et à la diffusion de documents scientifiques de niveau recherche, publiés ou non, émanant des établissements d'enseignement et de recherche français ou étrangers, des laboratoires publics ou privés. 


\title{
Assessment of ZDES for WMLES of turbulent boundary layers with pressure gradient and mild flow separation
}

\author{
Jaime Vaquero*, Nicolas Renard ${ }^{\dagger}$ and Sébastien Deck \\ ONERA - The French Aerospace Lab, F-92190 Meudon, France
}

Two test cases for the assessment of ZDES mode 3 (WMLES approach) in both pressure gradient conditions and mild boundary layer separation are presented. For both of them, the confinement effects of side wall boundary layers from the reference experiments are non negligible and they are taken into account in two dimensional simulations by means of a top wall geometry modification. A better agreement in the pressure coefficient with experimental data results from this manipulation. Results from the first test case evidence the advantage of resolving turbulence. A more physical flow is predicted in such a case and more in depth analysis of turbulence is possible, for instance spectral analysis as presented in this work. RANS results for the boundary layer separation case are presented showing the improvement on the pressure coefficient prediction thanks to the top wall geometry modification. At the time of the redaction of this manuscript, ZDES results for the boundary layer separation case are not available yet. However, the initial run of the ZDES simulation is very encouraging and suggests that a significant improvement over RANS predictions might be achieved.

\section{Nomenclature}

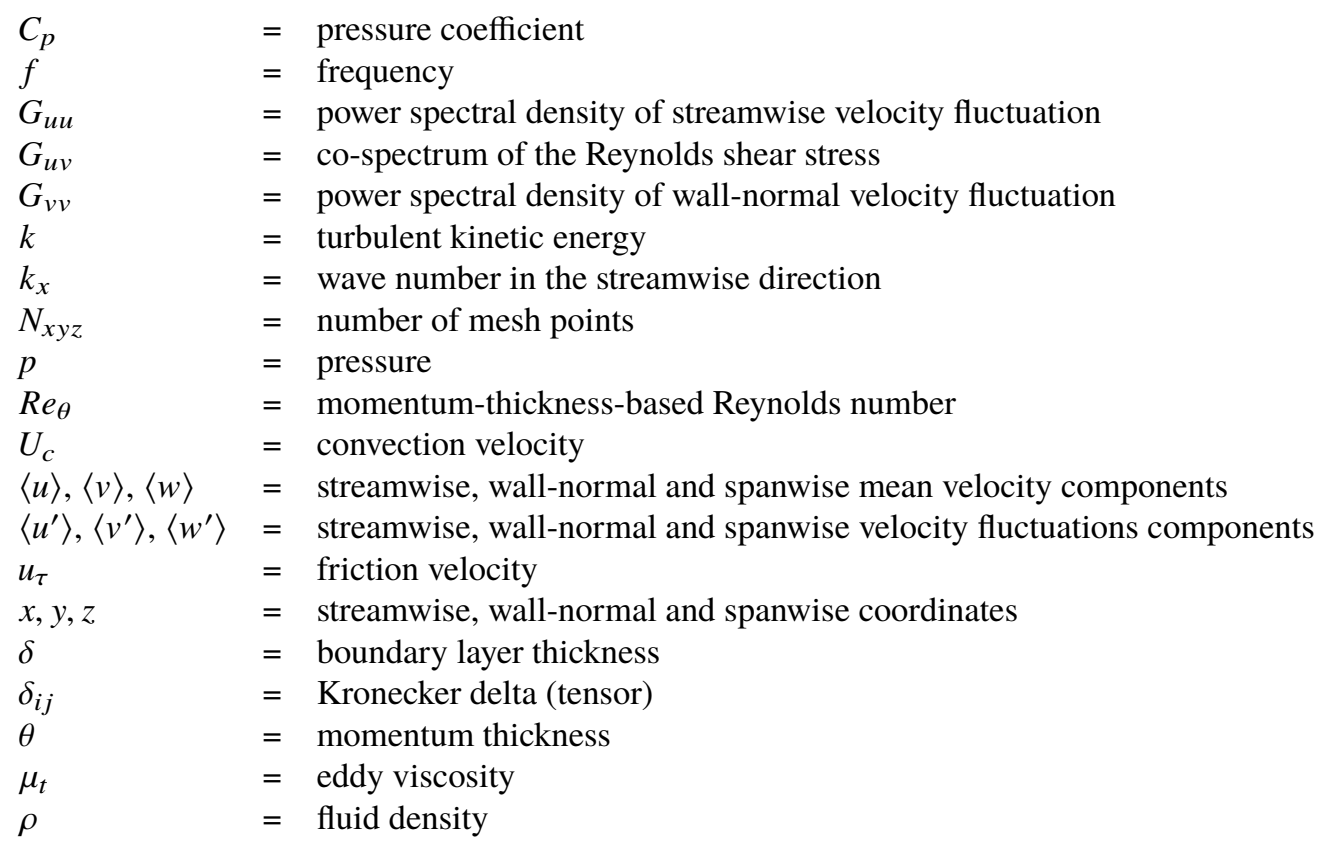

\section{Introduction}

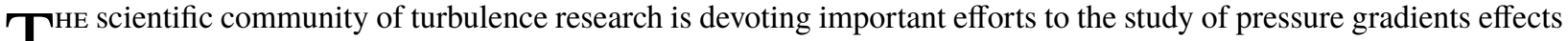
1 on turbulent boundary layers and separation. A better understanding of these would lead to a significant improvement

\footnotetext{
*Ph.D. Student, Aerodynamics, Aeroelasticity, Acoustics Department, jaime.vaquero@onera.fr

†Senior Research Scientist, Aerodynamics, Aeroelasticity, Acoustics Department, nicolas.renard@onera.fr

־Senior Research Scientist, Aerodynamics, Aeroelasticity, Acoustics Department, sebastien.deck@onera.fr
} 
of performance in many industrial devices. Nowadays, numerical simulations are essential in fluid mechanics research and broaden the research possibilities by complementing experimental studies. However, when considering turbulence numerical simulations there are still important practical limitations. A turbulent flow exhibits a wide range of turbulent scales and the separation between the smallest ones and the largest ones increases when so does the Reynolds number [1, 2]. At typical Reynolds number of industrial applications, the separation of scales is so important that directly solving all the flow structures (Direct Numerical Simulation, DNS) requires extremely fine meshes that, together with the huge number of time steps for statistical convergence, results in non-affordable simulations despite the most advanced high performance computation resources. In particular, in the first test-case presented later in this work [3], a DNS would require (at the considered Reynolds number) $N_{x y z}=50 \times 10^{9}$ points whereas the approach used in the present work requires $N_{x y z}=46 \times 10^{6}$. As a consequence, avoiding solving some (or all) of the turbulent scales is a widely used alternative. Reynolds-Averaged Navier Stokes equations allow to solve the mean field and require a closure for the Reynolds stress term in the momentum equation. This is known as RANS approach. Another approach extensively used is the Large Eddy Simulation (LES) which aims at solving only the energetic scales, and modeling the dissipative (small) scales by means of a subgrid-scale model (SGS model). Nevertheless, in wall-bounded turbulent flows, the relaxation in the mesh size is not very important between Wall-Resolved LES (WRLES) and DNS [4]. A bigger reduction of the computational effort may be obtained by modeling the near wall region using an approach called Wall- Modeled LES (WMLES) in contrast to WRLES. Such approach allows to decrease the number of points in the inner region of the boundary layer resulting in a significant lessening of the total number of points [4, 5]. Indeed, according to Deck et al. [4], the number of points increases as $N_{x y z} \sim R e_{\theta}^{2.4}$ for a DNS, $N_{x y z} \sim R e_{\theta}^{2.17}$ for a WRLES and $N_{x y z} \sim R e_{\theta}^{1.17}$ for a WMLES, where $R e_{\theta}$ is the Reynolds number based on the momentum thickness. In other words, higher Reynolds numbers at an affordable cost are achievable by means of WMLES, compared to WRLES or DNS. Among the different methods of using WMLES (which are not discussed in this work), the mode 3 of the Zonal Detached Eddy Simulation approach (ZDES) is used in this study, which is a hybrid RANS/LES approach and it has been validated for zero-pressure-gradient turbulent boundary layers [4]. Most studies related to pressure gradient boundary layers or turbulent boundary layer separation are made either experimentally or numerically using DNS or WRLES [6-10]. However, due to the computational cost, numerical studies are in general limited in terms of Reynolds number. In the present work, two high-Reynolds number test-cases are considered: the first one for pressure gradient effects on turbulent boundary layers and the second one for turbulent boundary layer mild separation. Both test cases provide Reynolds numbers great enough to illustrate in terms of computational cost the interest of the ZDES mode 3, which is again proved in the results obtained for the first test case, making this approach very promising for the second one (for which ZDES mode 3 results are not available yet at the time of elaboration of the present manuscript).

This manuscript is structured as follows. First, the turbulence modeling techniques used are presented and in particular the ZDES mode 3 is detailed. Then the first test-case devoted to the effects of pressure gradients is presented and results are commented. And finally the second test-case for the study of boundary layer separation is introduced.

\section{Turbulence modeling}

\section{A. RANS models}

Several RANS models are considered in the present study, mainly the Spalart-Allmaras [11], the $k-\omega$ SST from Menter [12] (which are first order models) and the Reynolds Stress Model (RSM) SSG-LRR- $\omega$ [13-15]. First order models add equations of transport for turbulent variables used to link the Reynolds stress tensor to the mean flow variables. This closure is in many cases (but not always) given by the Boussinesq's hypothesis which in an incompressible case writes:

$$
-\rho\left\langle u_{i}^{\prime} u_{j}^{\prime}\right\rangle=2 \mu_{t}\left\langle S_{i j}\right\rangle-\frac{2}{3} \rho k \delta_{i j}, \quad\left\langle S_{i j}\right\rangle=\frac{1}{2}\left(\frac{\partial\left\langle u_{i}\right\rangle}{\partial x_{j}}+\frac{\partial\left\langle u_{j}\right\rangle}{\partial x_{i}}\right)
$$

where $\langle\bullet\rangle$ denotes Reynolds average, $\rho$ is the density, $u_{i}^{\prime}$ is the $i^{\text {th }}$ component of the fluctuating velocity, $\mu_{t}$ the eddy viscosity, $k$ the turbulent kinetic energy and $\delta_{i j}$ the Kronecker delta (tensor). In the case of second order models, the transport equation is considered for the Reynolds stresses and writes

$$
\frac{\partial}{\partial t}\left\langle u_{i}^{\prime} u_{j}^{\prime}\right\rangle+\left\langle u_{l}\right\rangle \frac{\partial}{\partial x_{l}}\left\langle u_{i}^{\prime} u_{j}^{\prime}\right\rangle=\mathcal{P}_{i j}+\mathcal{R}_{i j}-\frac{\partial}{\partial x_{l}} \mathcal{T}_{l i j}-\varepsilon_{i j}
$$


(I)

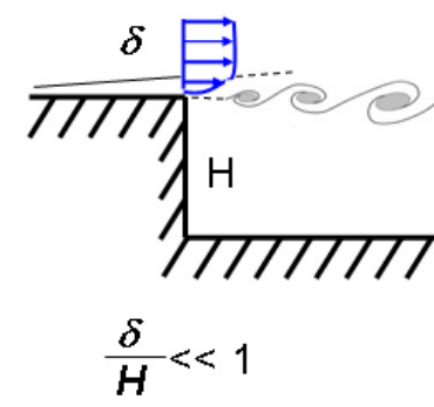

(II)

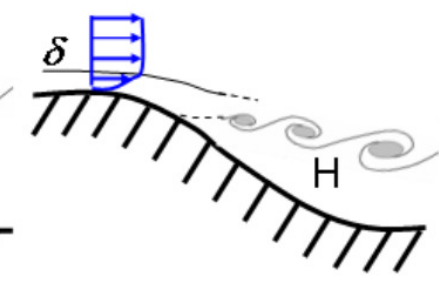

$\frac{\delta}{H} \ll 1$
(III)

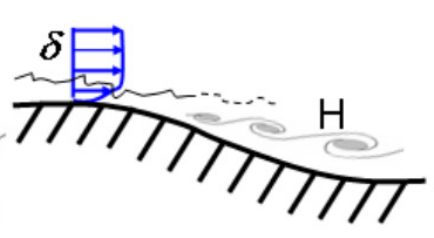

Fig. 1 Classification of typical flow types for the ZDES approach. I: separation fixed by the geometry, II: separation induced by a pressure gradient on a curved surface, and III: separation strongly influenced by the dynamics of the incoming boundary layer.

Due to the symmetry of the Reynolds stress tensor, the additional number of equations to solve are one for each Reynolds stress (which makes 6) and an additional one for a given turbulent variable, which my be the turbulent kinetic energy disspiation $\varepsilon$ or the specific dissipation rate $\omega[1]$. In equation $(2), \mathcal{P}_{i j}$ is the production tensor, $\mathcal{R}_{i j}$ is the pressure-rate-of-strain tensor, $\mathcal{T}_{l i j}$ the Reynolds stress flux and $\varepsilon_{i j}$ the dissipation tensor.

\section{B. ZDES mode 3}

The Zonal Detached Eddy Simulation is a hybrid RANS/LES approach that allows to treat a given problem by means of three different operating modes depending on the flow configuration as illustrated in figure 1 . This approach has been developed since the early 2000s and it is described in the work of Deck [16]. Among the three modes, the mode 3 is used in the present study since it corresponds to a WMLES approach (although it may also be used for WRLES [17]). A detailed description of the method is presented in the following.

The mode 3 of the ZDES uses the turbulence model of Spalart and Allmaras [11]. In the inner part of the boundary layer, a RANS approach is performed whereas in the outer part and in the outside of the boundary layer a LES approach is used. The same turbulence model is used both for RANS and as a SGS model for LES. The separation between the RANS region and the LES region is given by an interface (in particular by its wall distance $d_{w}^{\text {int }}$ ) which is explicitly provided by the user. The characteristic length of the turbulence model $\tilde{d}_{\mathrm{ZDES}}^{\mathrm{III}}$, once the position of the interface defined, is given by

$$
\tilde{d}_{\mathrm{ZDES}}^{\mathrm{III}}=\left\{\begin{array}{cl}
d_{w} & \text { if } d_{w}<d_{w}^{\text {interface }} \\
\min \left(d_{w}, C_{\mathrm{DES}} \Delta_{\mathrm{vol}}\right) & \text { if } d_{w} \geq d_{w}^{\text {interface }}
\end{array}\right.
$$

where $C_{\mathrm{DES}}=0.65$ and $\Delta_{\mathrm{vol}}=\mathcal{V}^{1 / 3}$ being $\mathrm{V}$ the cell volume. Other slight modifications of the model are also made for a proper behavior and the reader may find them in [16]. Besides, a recent improvement of the method has been made by Renard and Deck [18] which smoothens the binary transition from RANS to LES near the RANS/LES interface. A continuous function $f_{\delta}$ between 0 and 1 with also continuous derivatives is included in the new expression of $\tilde{d}_{\mathrm{ZDES}}^{\text {III }}$ :

$$
\tilde{d}_{\mathrm{ZDES}}^{\mathrm{III}}=\left(1-f_{\delta}\right) \cdot d_{w}+f_{\delta} \cdot \min \left(d_{w}, C_{\mathrm{DES}} \Delta_{\mathrm{vol}}\right)
$$

as well as in the model functions $f_{v 1}, f_{v 2}$ and $f_{w}$, which, with the mentioned modifications [16], they become:

$$
\begin{aligned}
f_{v 1}^{\mathrm{III}} & =\left(1-f_{\delta}\right) \cdot f_{v 1}+f_{\delta} \\
f_{v 2}^{\mathrm{III}} & =\left(1-f_{\delta}\right) \cdot f_{v 2} \\
f_{w}^{\mathrm{III}} & =\left(1-f_{\delta}\right) \cdot f_{w}+f_{\delta} .
\end{aligned}
$$




\section{Inflow boundary conditions and history effects}

Numerical simulations in which turbulence is either partially or totally resolved, as in the case of ZDES mode 3, require unsteady boundary conditions. Among the several ways to introduce turbulence in a computational domain (see Deck et al. [19]), the Synthetic Eddy Method (SEM) is considered in the present study. Briefly, this consists in injecting fluctuations to a mean flow that are intended to mimic turbulence. Since those fluctuations are not perfectly realistic, they require some amount of time (and therefore convection in the domain) until they become proper turbulence of the problem studied.

The behavior of SEM for studies of zero-pressure-gradient turbulent boundary layers is very satisfactory Deck et al. [19]. Despite the numerous studies of the effects of upstream perturbations on turbulent boundary layers, some discrepancies where found when it comes to adverse pressure gradient conditions that led the authors to a prior study in order to identify whether disturbances of the boundary layer in such conditions would eventually disappear or, contrary to that, increase [20]. The results show that convergence to a reference state requires important distances of the order of $10^{4}$ and $10^{2}$ times the initial boundary layer thickness for laminar and turbulent boundary layers respectively. Besides, it was observed that for all of the favorable pressure gradient cases and the moderate adverse pressure gradient ones, convergence was always obtained. Therefore, this is a satisfactory result that allows to use the unsteady boundary conditions in the same way as for previous zero-pressure-gradient studies [19] since the possible defects of the inflow condition will gradually vanish after a sufficient convection distance.

\section{Numerical methods}

Different modeling approaches are used in this work, therefore different numerical methods are suitable. Differences are found between RANS simulations (either first or second order models) and ZDES simulations. Indeed the flow solved is intrinsically different between both approaches and the numerical methods suitable for one are not the same as those for the other. Besides, it must be pointed out that a different solver has been used for the $k-\omega$ SST and the RSM simulations although the same numerics are used than for the others RANS simulations.

In the case of RANS simulations, the finite volume method with Roe's scheme is used for spatial discretization together with a MUSCL approach (Monotonic Upstream Scheme for Conservation Laws) for flux reconstruction at the cell faces. A pseudotemporal integration is done by virtue of an implicit Euler's scheme. Simulations with the Spalart-Allmaras model are made using the in-house ONERA solver FLU3M, and those using the $k-\omega$ SST model and the RSM model are made with the ONERA industrial solver elsA. In order to avoid uncertainty in the results obtained coming from the fact of using different solvers, comparisons between the two solvers using the Spalart-Allmaras model and the same numerical schemes have been made (not shown) giving a perfect agreement.

Regarding the ZDES approach, simulations are performed with the solver FLU3M. The definition of the numerical methods employed is slightly more complex. In fact, due to the zonal feature of the method, the ZDES mode 3 is considered only for the boundary layer of interest, which is the one developing over the bottom wall (in which experimental measurements have been recorded in both test cases [21, 22]). At the top wall a full unsteady RANS approach (URANS) with the Spalart-Allmaras model is retained. Thus, modeling effects are only impacting the boundary layer of interest when comparing to full RANS simulations. Besides, this is the reason why in figure 3 turbulent structures in the schlieren contour are solely observable in the bottom-wall boundary layer. This illustrates the capacity of ZDES to focus only on the region of interest while resorting to RANS for the rest of the flow at a reduced computational cost, contrary to typical full WMLES strategies. In the (approximate) bottom half domain, where the ZDES mode 3 is applied, the spatial scheme is the AUSM + (P) (proposed by Liou [23] and modified as in [24] for dissipation reduction purposes). In the upper domain, the AUSM + (P) scheme is employed (without the modification of Mary and Sagaut [24], contrary to Roe's scheme used in full RANS simulations. The reason for this change is based on numerical schemes compatibility between the ZDES mode 3 region and the RANS region in that simulation.

\section{Effects of pressure gradients on turbulent boundary layers: description of the problem studied}

The experimental work of Cuvier et al. [21] (for the lowest free stream velocity) has been chosen for numerical reproduction due to several aspects. On the one hand, the Reynolds number of the problem is representative of applied aerodynamics problems where hybrid RANS/LES approaches are suitable because the numerical effort for a DNS or WRLES would be excessive. Indeed, reaching this Reynolds number of $R e_{\theta} \approx 13000$ (where $\theta$ is the boundary layer momentum thickness) would require $50 \times 10^{9}$ and $3 \times 10^{9}$ grid points respectively for DNS and WRLES. In the case of 

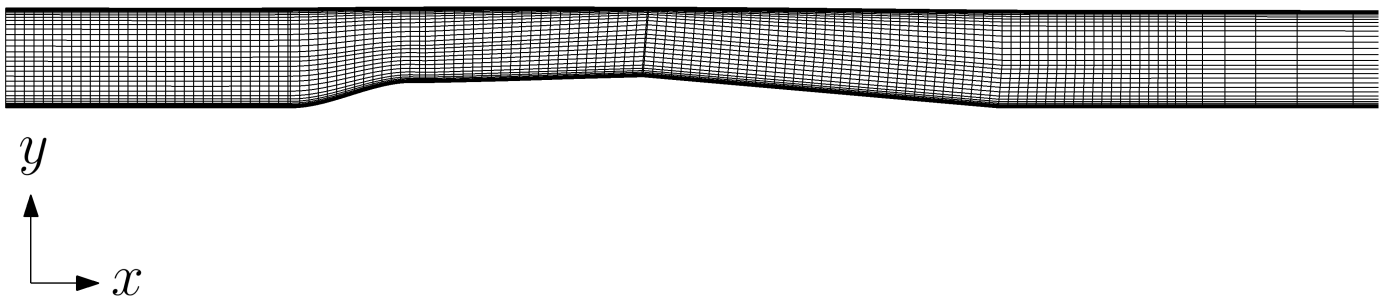

Fig. 2 Lateral view of the mesh employed for the ZDES simulation, where only one point over eight is shown in the $x$ and $y$ directions. In each direction, the number of points is $N_{x}=1050, N_{y}=273$ and $N_{z}=160$.

ZDES mode 3 instead, the number of mesh points is significantly reduced down to $46 \times 10^{6}$ (around a thousand and a hundred times less points). Given the computational cost of such simulations, some decisions have been made to decrease the number of points as much as possible. In particular, the side-walls boundary layers are not solved (and periodicity is imposed in the span boundaries) but their contraction effect (which is non-negligible due to the size of the boundary layer with respect to the span of the wind tunnel) is taken into account by means of a modification of the top wall geometry as detailed in [3].

A view of the mesh and the computational domain for the ZDES simulation is presented in figure2 2 in which the flow goes from left to right and the mesh resolution is taken as $\Delta x^{+}=200, \Delta z^{+}=100$ and $\Delta y_{w}^{+}=1$ (the symbol '+'refers to viscous scales). In the case of the RANS simulations, the mesh spacings are the same and the only difference is that the inlet of the domain is placed farther upstream. More precisely, for the latter the inlet is placed at $x / L=-2.01$ whereas in the ZDES computation it is placed at $x / L=6.6$, where $L$ is the real height of the wind tunnel. It is reminded that for the ZDES simulation, unsteady boundary conditions (SEM) are used which already give a boundary layer profile at the inlet and therefore this allows to place the inlet farther downstream than in RANS computations where boundary layer develops from an uniform inlet boundary condition. Besides, the SEM requires a boundary layer profile which is obtained from the RANS computations, which is the reason why the computational domain is longer in RANS computations. It is also specified that a dynamic forcing method is used to reduce the relaxation length of fluctuations injected by the SEM [19, 25]. For the ZDES simulation, the RANS/LES interface is placed so that $d_{w}^{\text {int }}=0.1 \delta$ where $\delta$ is the boundary layer thickness defined as $\langle u\rangle_{(y=\delta)}=0.99 U_{e}$, being $U_{e}$ the external streamwise velocity.

\section{Results}

\section{A. Instantaneous field}

The instantaneous field obtained from the ZDES mode 3 simulation is presented at first. Figure 3 shows an isosurface of $Q$-criterion together with a numerical schlieren (norm of density gradient). As observed in this figure, an important part of turbulence in the boundary layer is resolved and the hairpin type structure is observable which is a typical structure found in turbulent boundary layers [26]. As already mentioned in a previous section, the schlieren contours do not show any turbulent fluctuation in the upper boundary layer due to the zonal feature of the ZDES approach allowing to use a full RANS model for this boundary layer. However, a smooth gradient is still observable resulting from temperature increase as a consequence of friction losses.

\section{B. Mean field}

Profiles of the mean velocity and the Reynolds shear stress are presented in figure 4 for all the models considered (Spalart-Allmaras, $k-\omega$ SST, SSG-LRR- $\omega$ and ZDES mode 3 ) at $x / L=10.73$. This station is placed right upstream of the favorable-pressure-gradient flat plate and it is chosen due to the unusual shape of the profiles compared to zero-pressure-gradient ones. Indeed, the experimental data shows a velocity excess in the mean velocity profile at $y=0.15 \delta$ as well as an external peak in the Reynolds shear stress profile. It is observed that, among the models presented, the Spalart-Allmaras is the least accurate the most and is not able to reproduce the external peak. Better results, yet perfectible, are given by the $k-\omega$ SST model which also misses the external peak. The RSM model is more accurate in the mean velocity profile and it is able to predict the external peak of the Reynolds shear stress, however the 


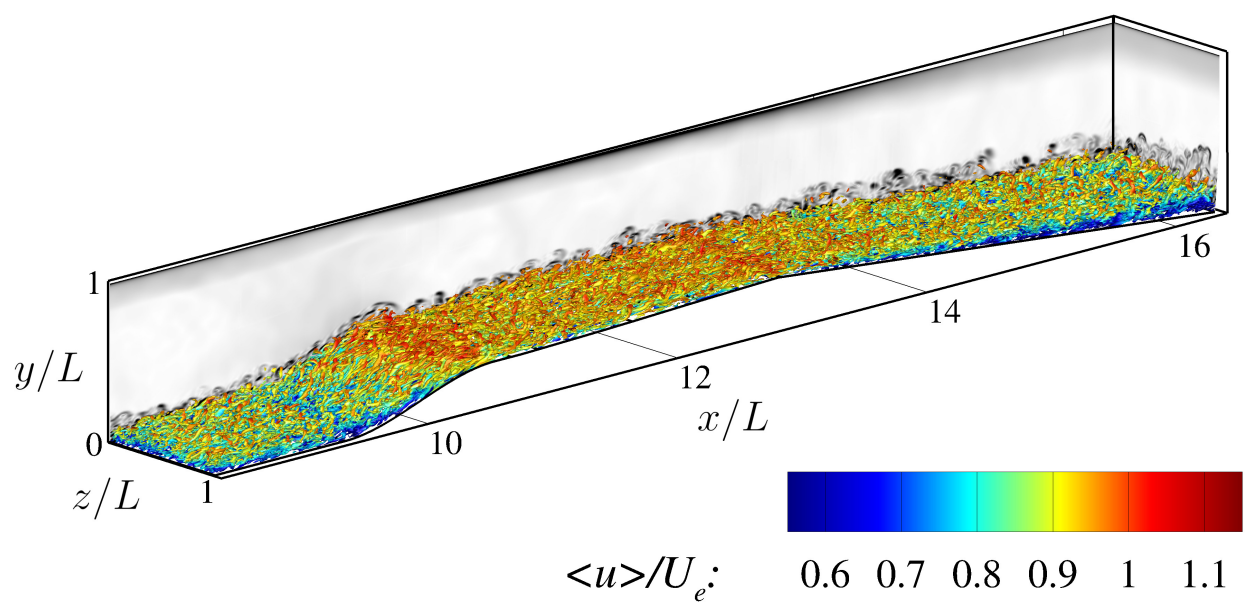

Fig. 3 Isosurface of the $Q$-criterion for $Q=0.14\left(\frac{U_{0}}{\delta_{0}}\right)^{2}$ together with numerical schlieren contours.
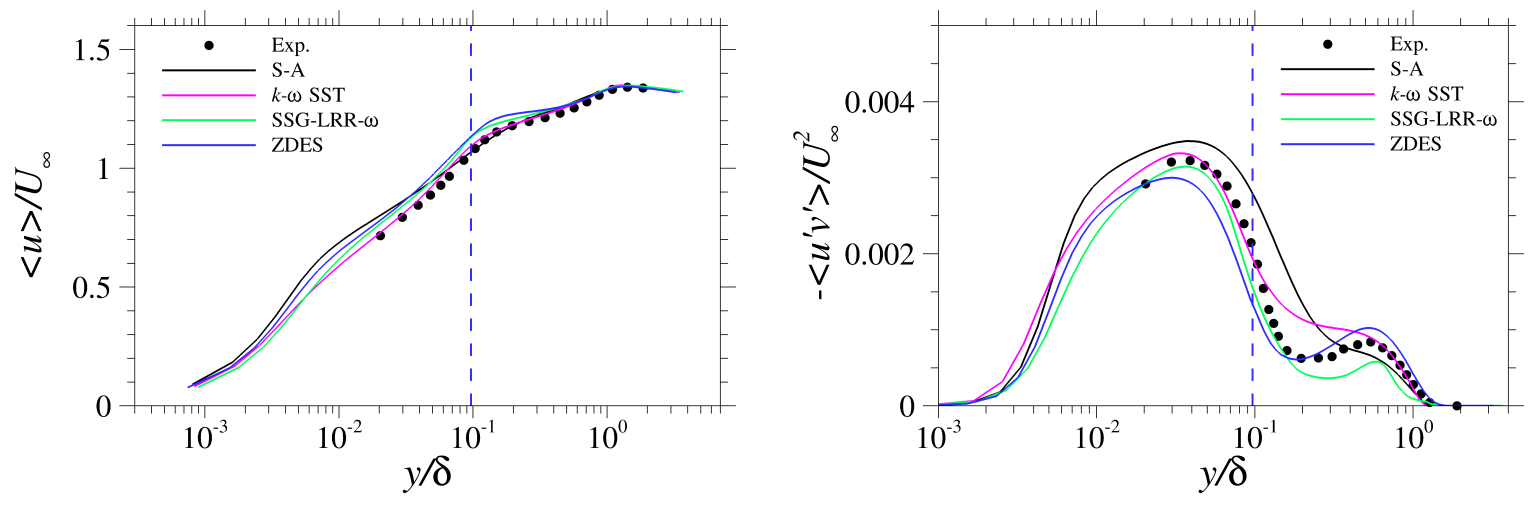

Fig. 4 Profiles of mean velocity (left) and Reynolds shear stress (right) at $x / L=10.73$. 

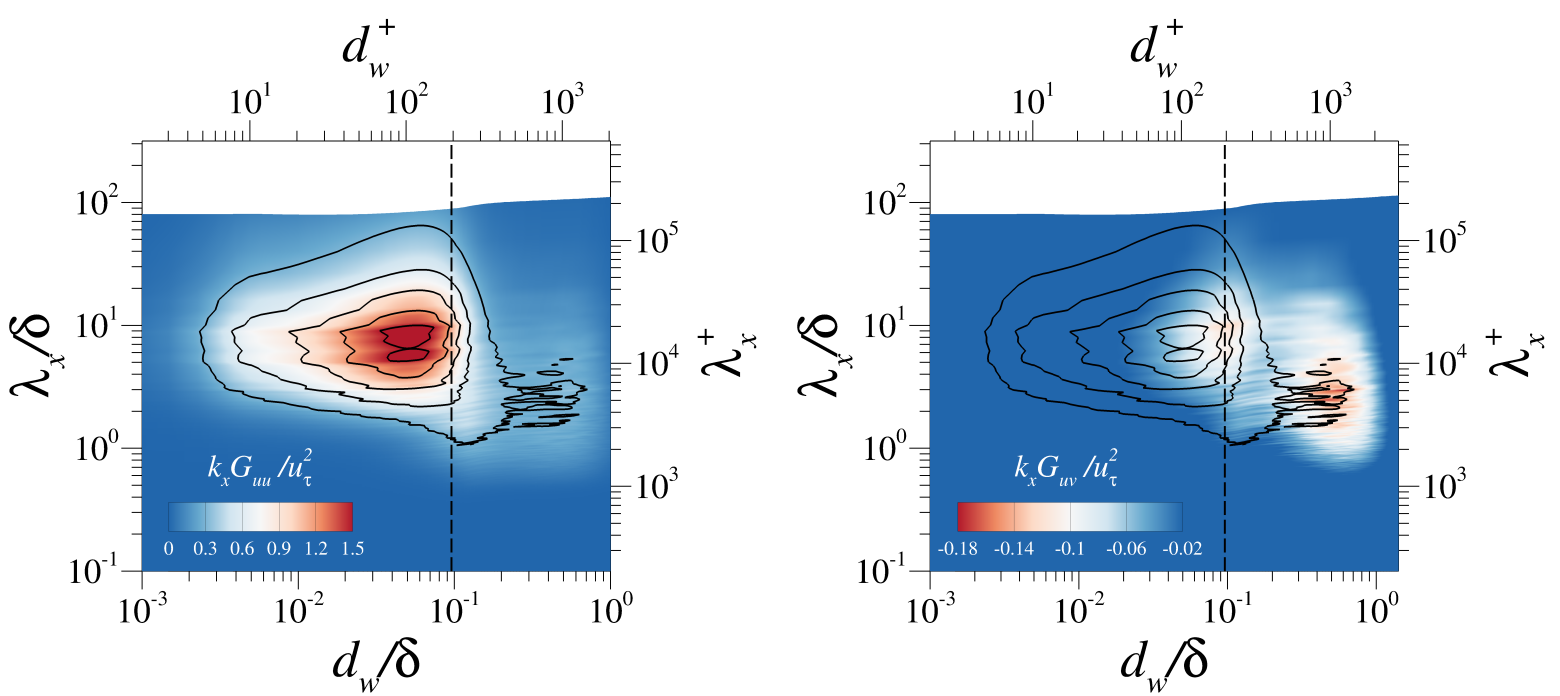

Fig. 5 Left: Premultiplied power spectral density (PSD) of streamwise velocity fluctuations $k_{x} G_{u u} / u_{\tau}^{2}$. Right: premultiplied co-spectrum of the Reynolds shear stress $k_{x} G_{u v} / u_{\tau}^{2}$. Black contours represent $k_{x} G_{u u} / u_{\tau}^{2}$. Dashed lines correspond to the position of the RANS/LES interface.

shape of this profile as well as its levels are more accurately represented by the ZDES mode 3. More profiles at different stations are provided in Vaquero et al. [3].

\section{Spectral assessment of Reynolds stresses}

The satisfactory results from the ZDES mode 3 at the station presented in the previous section together with the feature of resolving part of the turbulence have encouraged to perform a spectral analysis of turbulence at that same station. The power spectral density (PSD) of the streamwise velocity fluctuation and the premultiplied co-spectrum of the Reynolds shear stress normalized as in equations (6) and (7) are given in figure 5. It is interesting to notice a second region of energetic content in the streamwise velocity which is significantly more noticeable in the Reynolds shear stress and whose location corresponds to that of the external peak of the Reynolds shear stress profiles. This energy is associated to structures of about $2 \delta$ to $3 \delta$ long and therefore these structures are likely responsible for these unusual features found in the mean velocity profile and the Reynolds shear stress profiles.

$$
\begin{aligned}
& \left\langle u^{\prime 2}\right\rangle=\int_{0}^{+\infty} G_{u u}\left(k_{x}\right) \mathrm{d} k_{x}=\int_{-\infty}^{+\infty} k_{x} G_{u u}\left(k_{x}\right) \mathrm{d}\left(\ln \left(k_{x}\right)\right) \\
& \left\langle u^{\prime} v^{\prime}\right\rangle=\int_{0}^{+\infty} G_{u v}\left(k_{x}\right) \mathrm{d} k_{x}=\int_{-\infty}^{+\infty} k_{x} G_{u v}\left(k_{x}\right) \mathrm{d}\left(\ln \left(k_{x}\right)\right)
\end{aligned}
$$

\section{Evidence of two separated turbulent sites}

The previous spectral analysis has allowed to identify that turbulent structures of wavelength $\lambda_{x}$ between $2 \delta$ and $3 \delta$ strongly contribute to the dynamics of the outer peak observed in the Reynolds shear stress profile. In order to try to better comprehend the mechanisms lying under this peak, the PSD of the production term of the Reynolds shear stress as well as a scale-dependent convection velocity are analyzed. For a flat plate boundary layer, the production term of $-\left\langle u^{\prime} v^{\prime}\right\rangle$ is given by (see [1])

$$
\mathcal{P}_{-\left\langle u^{\prime} v^{\prime}\right\rangle}=\left\langle v^{\prime 2}\right\rangle \frac{\partial\langle u\rangle}{\partial y} .
$$

The power spectral density is obtained from $G_{v v} \partial\langle u\rangle / \partial y$, where $G_{v v}$ is the PSD of $\left\langle v^{\prime 2}\right\rangle$ which is defined analogously to $G_{u u}$ in equation (6). The PSD of $\mathcal{P}_{-\left\langle u^{\prime} v^{\prime}\right\rangle}$ is displayed in figure 6, where two different sites of Reynolds stress production are identified. Higher energy levels are present in the outer site, for which structures of $\lambda_{x}=2 \delta-3 \delta$ are mainly responsible. However, at lower levels, longer turbulent structures also contribute to the production of $\left\langle u^{\prime} v^{\prime}\right\rangle$ in this site, 


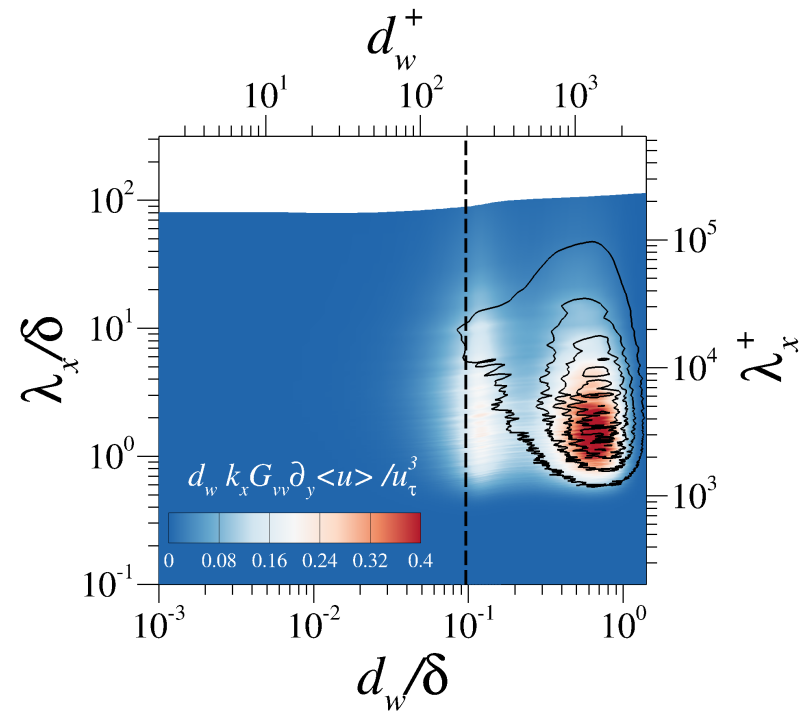

Fig. 6 Premultiplied power spectral density (PSD) of Reynolds shear stress production term (premultiplied both by the wall distance $d_{w}$ and the wave number $\left.k_{x}\right)$. Black contours on the left: $d_{w} k_{x} G_{u v} /\left(u_{\tau}^{2} \delta\right)$.

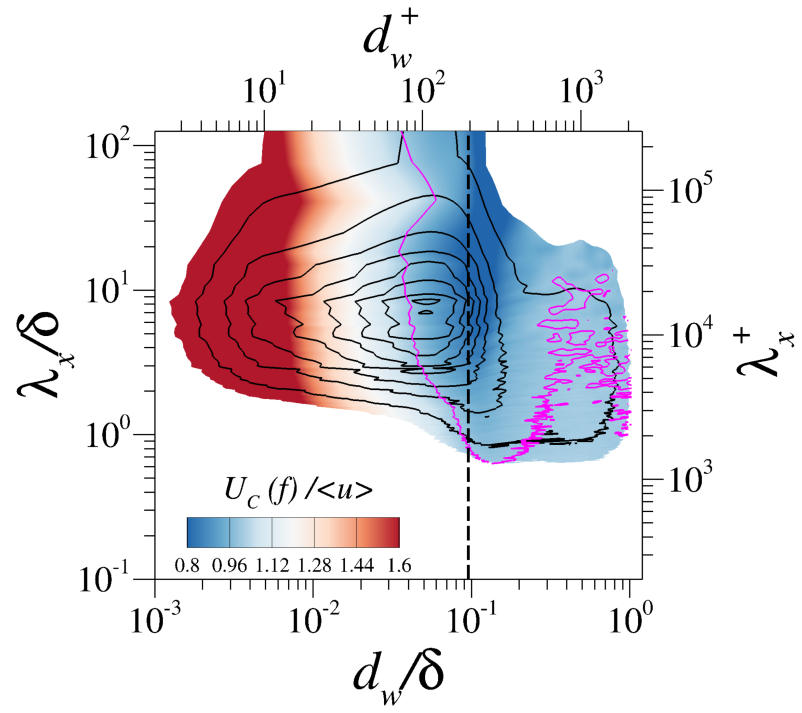

Fig. 7 Local frequency-dependent convection velocity normalised by the local mean velocity. Purple contours: loci of $U_{c}(f)=\langle u\rangle$. Black contours on the right: $k_{x} G_{u u} / u_{\tau}^{2}$. Dashed lines correspond to the position of the RANS/LES interface. 


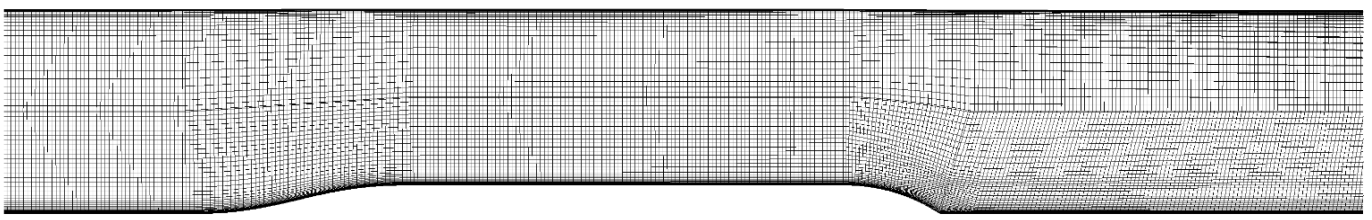

Fig. 8 Lateral view of the mesh employed for the RANS simulation, where only one point over four is shown in the $x$ (horizontal) and $y$ (vertical) directions. The real domain is larger in the streamwise direction. Flow goes from left to right.

that reach wavelengths up to $\lambda_{x}=8 \delta$. It is interesting to notice that the co-spectrum of $\left\langle u^{\prime} v^{\prime}\right\rangle$ coincides fairly well with the PSD of $\mathcal{P}_{-\left\langle u^{\prime} v^{\prime}\right\rangle}$ in the outer site, but only at large scales for the site closer to the wall. The production in this site for scales below $\lambda_{x} \approx 5 \delta$ is compensated up to $25 \%$ by the mean-flow convection $\left(=-\left[\langle u\rangle \partial_{x}\left(-\left\langle u^{\prime} v^{\prime}\right\rangle\right)+\langle v\rangle \partial_{y}\left(-\left\langle u^{\prime} v^{\prime}\right\rangle\right)\right]\right)$ term of the Reynolds shear stress transport equation, contrary to zero-pressure-gradient turbulent boundary layers for which the mean-flow convection contribution is negligible [1]. The remaining part of the production contribution is likely compensated by the pressure term, as in zero-pressure-gradient turbulent boundary layers [1].

The convection velocity of $u^{\prime}$ dependent on the frequency, $U_{c}(f)$, is plotted in figure 7 normalized by the mean velocity $\langle u\rangle . U_{c}(f)$ is computed according to the work of Renard and Deck [27], and it provides a convection velocity at each position of the boundary layer for the different turbulent scales involved, since Taylor's hypothesis allows to link the frequency of the signal $f$ and the wavelength $\lambda_{x}$. As observed in figure 7, the convection velocity close to the wall is in overall significantly greater than the local mean velocity, which is in accordance with the results of Renard and Deck [27]. Besides, Renard and Deck [27] found that near the three energetic sites in the PSD of $\left\langle u^{\prime 2}\right\rangle$, the convection velocity was quite close to the local mean velocity (analogy with the critical layer stability concept). Such a result is also observed in figure 7 for both the inner $\left(d_{w} \approx 0.05 \delta\right)$ and outer $\left(d_{w} \approx 0.4 \delta\right)$ sites of $G_{u u}$ obtained with ZDES mode 3. Therefore, the outer site observed in figures 5,6 and 7 , and responsible for the outer peak in the Reynolds shear stress, is a separated energetic site from the inner one, the latter being very close to the peak observed in zero-pressure-gradient turbulent boundary layers (see [4]). Moreover, the convection velocity of the outer site is about 1.5 times greater than that of the inner site.

\section{Application of ZDES mode 3 to a turbulent boundary layer with separation and reattachment}

\section{A. Test case for boundary layer separation}

The separation of a turbulent boundary layer predicted by means of the RANS approach is generally quite perfectible. It is proposed thus to use the ZDES mode 3 since an important part of turbulence is resolved instead of modeled at an affordable cost. The experimental study of Song and Eaton [22] is reproduced numerically. This work has been chosen mainly due to the Reynolds numbers presented and, in particular, the case $R_{\theta}=13200$ is considered numerically. As with the test case of the previous sections, such a Reynolds number is not affordable using DNS or WRLES, therefore making the ZDES mode 3 of particular interest for this test case. A view of the mesh is shown in figure 8 in order to illustrate the geometry of the problem.

This experiment has already been reproduced numerically, and in particular using WMLES at the same Reynolds number by Radhakrishnan et al. [28]. Their WMLES is performed by means of the first formulation of the Detached Eddy Simulation [29] (usually referred to as DES97) which is known to create a mismatch in the logarithmic region of the boundary layer profile due to the low levels of eddy viscosity not being compensated by resolved turbulence. The ZDES mode 3 is able to deal quite satisfactorily with the mentioned issue. Indeed it has been validated for zero-pressure-gradient turbulent boundary layers [4, 19]. Besides, it is worth pointing that the mesh employed in the WMLES simulations of Radhakrishnan et al. [28] is much coarser than what the meshing requirements for ZDES mode 3 would demand, even for the fine mesh in Radhakrishnan et al. [28]. 


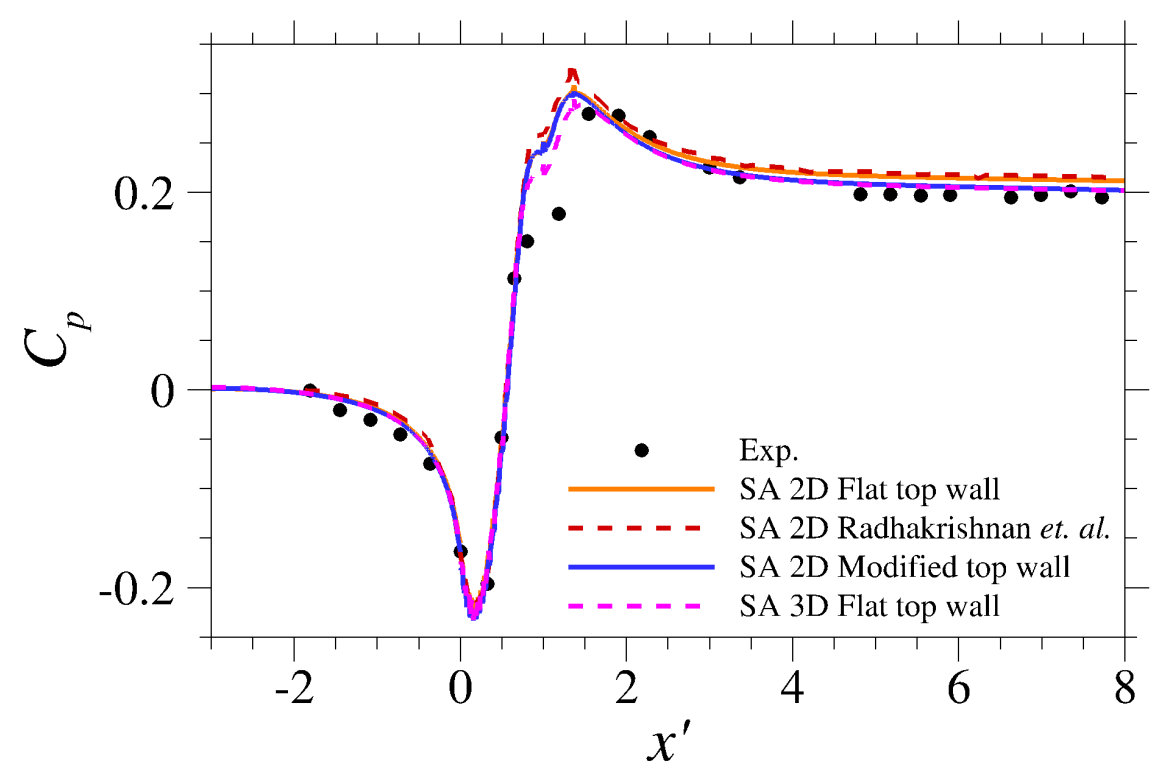

Fig. 9 Pressure coefficient evolution on the bottom wall.

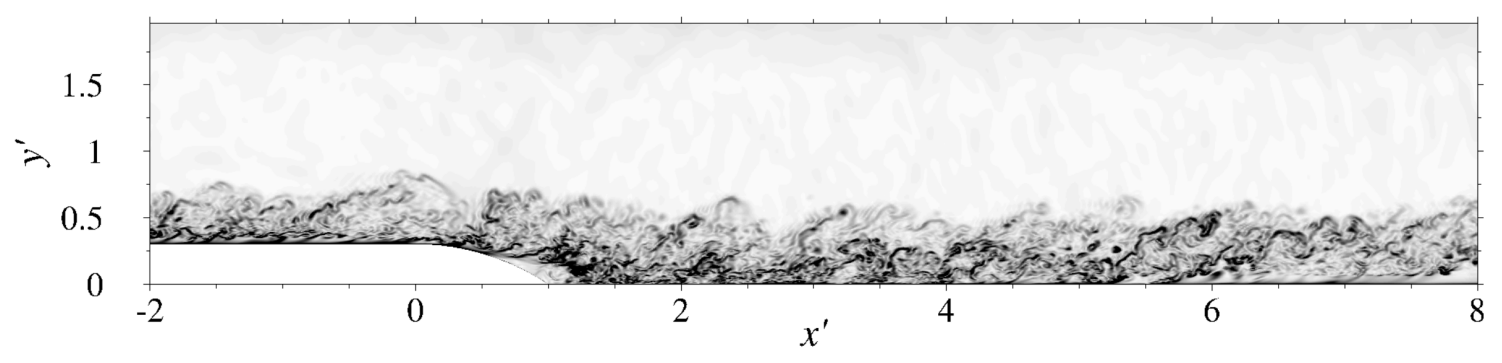

Fig. 10 Numerical schlieren (magnitude of density gradient) in the $(x, y)$ center plane.

\section{B. First results}

In this section, the first results obtained of RANS simulations with the Spalart-Allmaras turbulence model are presented. These are compared to those of Radhakrishnan et al. [28] showing an excellent agreement when the real wind tunnel top wall geometry is used (flat top wall). As it was the case in the simulations of previous sections, confinement effects from side walls boundary layers have again been found to be non negligible. Indeed, all of the models in the work of Radhakrishnan et al. [28] show a small over estimation of the pressure coefficient in the recovery region. Therefore, the same procedure as in the previous case (so the same as described in [3]) has been performed and the new geometry of the top wall allows for a more accurate prediction of the pressure coefficient (9). The pressure coefficient is defined as:

$$
C_{p}=\frac{p-p\left(x^{\prime}=-1.81\right)}{\frac{1}{2} \rho_{\text {ref }} U_{r e f}^{2}}
$$

where $x^{\prime}=x / a$ and $a$ is the length of the round step, and the reference values are taken at $x^{\prime}=-2$. The results of the two-dimensional simulation with a modified top wall geometry are in good accordance with those of the three-dimensinal simulation (in which the side wall boundary layers are resolved) which justifies the use of this new geometry for a more reliable reproduction of the experiment by means of two-dimensional simulations.

A ZDES mode 3 simulation is being carried out at the moment of the elaboration of the present manuscript. Results will be provided in the future works, in which mean field profiles at different stations will be presented as well as spectral analysis at some relevant positions of the flow. The initial run of the simulation is very encouraging and suggests that ZDES might show a significant improvement over RANS models in this test case. A visualization of the unsteady flow 


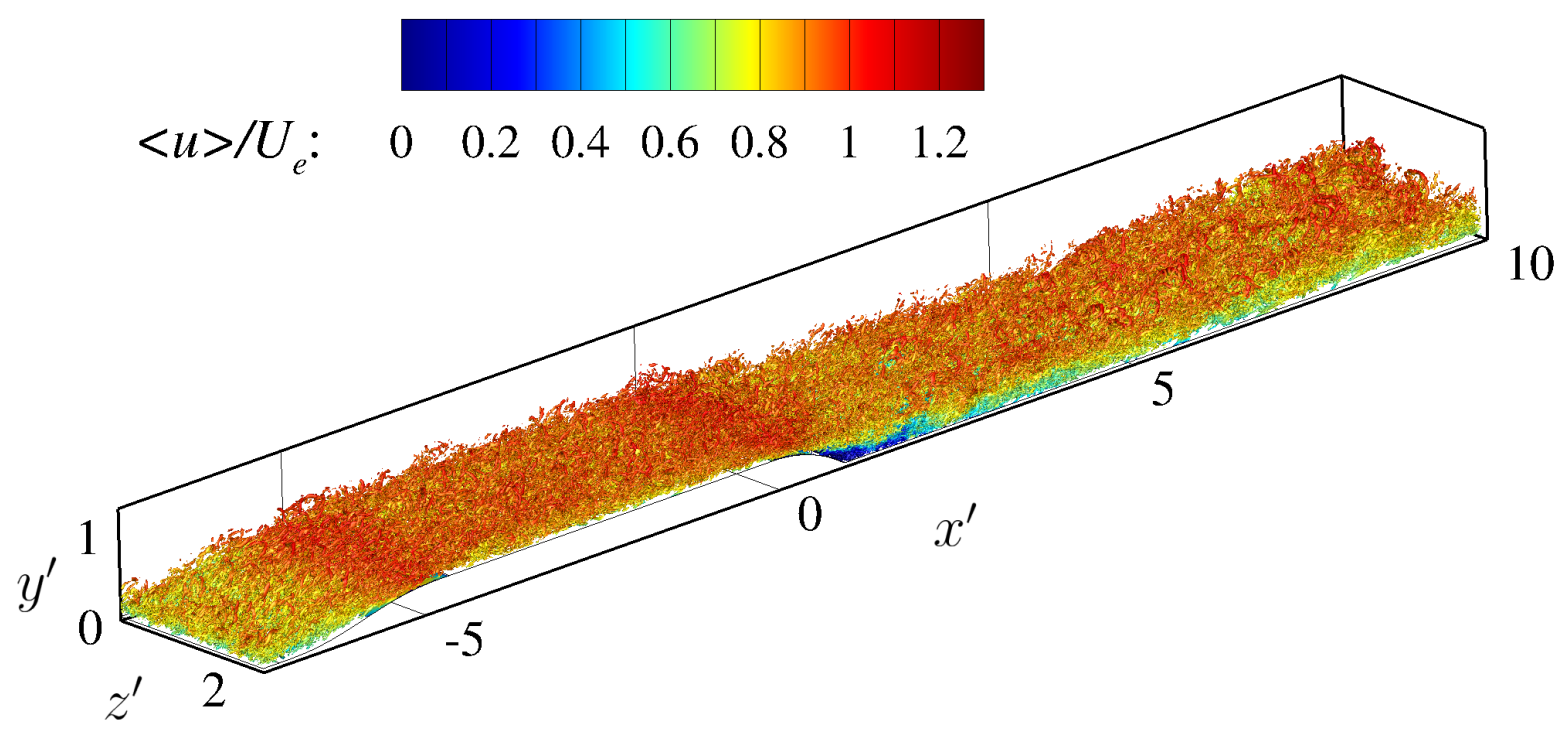

Fig. 11 Isosurface of the $Q$-criterion for $Q=0.19\left(\frac{U_{0}}{\delta_{0}}\right)^{2}$ colored by the streamwise velocity component $u$ (a reduced is shown for clarity purposes in the illustration).

field is already possible and presented in figures 10 and 11 by means of a numerical schlieren (norm of density gradient) and an iso-surface of $Q$-criterion respectively. Coordinates denoted with the $\bullet^{\prime}$ symbol are non-dimensionalized with the ramp length $a$.

\section{Conclusion}

Two test-cases are presented in this work for the assessment of ZDES mode 3 in pressure gradient conditions as well as in boundary layer separation. For both cases, a modification of the top wall is performed in order to take into account the confinement effects of side walls boundary layers in the mean flow as done in Vaquero et al. [3]. Thus, a better agreement of the pressure coefficient with experimental data is achieved. The first test-case points out the relevance of ZDES mode 3. Despite a greater numerical cost, resolving part of the turbulence allows to capture more physics of the flow therefore having a more reliable prediction of it. Besides, spectral analysis of turbulence is achievable which gives the possibility for more in depth turbulence studies. In particular in this case, the unusual features observed in the profiles of mean velocity and Reynolds shear stress have been related to turbulent structures of streamwise length from 2 to 3 times the boundary layer thickness. The spectral analysis of the production term of the Reynolds shear stress has confirmed the previous results, and two energetic sites have been evidenced at about $d_{w} \approx 0.12 \delta$ and $d_{w} \approx 0.7 \delta$. Moreover, it has been observed that in the inner peak, the production term does not result in significant levels of Reynolds shear stress, but it is rather compensated in part by the advection term, and very likely by the pressure term as well. Besides, the assessment of the scale-dependent local convection velocity has permitted to identify that the structures linked to the outer peak compose a separated energy site in the outer part of the boundary layer, and they are convected significantly faster than structures belonging to the inner site. Regarding the boundary layer separation case, it is only possible to give the first results from the RANS simulations as well as an insight of the unsteady flow field from the ZDES mode 3 simulation at the state of the work, but full results from the ZDES mode 3 simulation (statistics including spectral analysis) will be given in future work. 


\section{Acknowledgments}

The authors are very thankful to researchers from the LMFL for profitable discussions, especially C. Cuvier and J.-M. Foucaut. The authors are grateful as well to all the FLU3M and elsA codes developers. The thesis of J. Vaquero is partly funded by DGA (French defense procurement agency). It is also acknowledged the support from the framework of the ONERA research project FROTTEMENT.

\section{References}

[1] Pope, S. B., Turbulent Flows, Cambridge University Press, 2000. https://doi.org/10.1017/cbo9780511840531.

[2] Sagaut, P., Deck, S., and Terracol, M., Multiscale and Multiresolution Approaches in Turbulence, $2^{\text {nd }}$ ed., Imperial College Press, 2013. https://doi.org/10.1142/p878

[3] Vaquero, J., Renard, N., and Deck, S., "Advanced simulations of turbulent boundary layers under pressure-gradient conditions," Physics of Fluids, Vol. 31, No. 11, 2019, p. 115111. https://doi.org/10.1063/1.5126932. URL https://doi.org/10.1063/1.5126932

[4] Deck, S., Renard, N., Laraufie, R., and Sagaut, P., "Zonal Detached Eddy Simulation (ZDES) of a spatially developing flat plate turbulent boundary layer over the Reynolds number range $3150 \leq R_{\theta} \leq 14000$," Physics of Fluids, Vol. 26, 2014 , p. 025116. https://doi.org/10.1063/1.4866180

[5] Piomelli, U., "Wall-layer models for Large-Eddy Simulations," Progress in Aerospace Sciences, Vol. 44, 2008, pp. 437-446. https://doi.org/10.1016/j.paerosci.2008.06.001.

[6] Spalart, P. R., and Watmuff, J. H., "Experimental and numerical study of a turbulent boundary layer with pressure gradients," Journal of Fluid Mechanics, Vol. 249, 1993, p. 337. https://doi.org/10.1017/s002211209300120x

[7] Skote, M., Henningson, D., and Henkes, R., "Direct numerical simulation of self-similar turbulent boundary layers in adverse pressure gradient conditions," Flow, Turbulence and Combustion, Vol. 60, 1998, pp. 47-85. https://doi.org/10.1023/a: 1009934906108

[8] Skote, M., and Henningson, D. S., "Direct numerical simulation of a separated turbulent boundary layer," Journal of Fluid Mechanics, Vol. 471, 2002. https://doi.org/10.1017/s0022112002002173.

[9] Cheng, W., Pullin, D. I., and Samtaney, R., "Large-eddy simulation of separation and reattachment of a flat plate turbulent boundary layer," Journal of Fluid Mechanics, Vol. 785, 2015, pp. 78-108. https://doi.org/10.1017/jfm.2015.604

[10] Kitsios, V., Sekimoto, A., Atkinson, C., Sillero, J. A., Borrell, G., Gungor, A. G., Jiménez, J., and Soria, J., "Direct numerical simulation of a self-similar adverse pressure gradient turbulent boundary layer at the verge of separation," Journal of Fluid Mechanics, Vol. 829, 2017, pp. 392-419. https://doi.org/10.1017/jfm.2017.549.

[11] Spalart, P., and Allmaras, S., "A one-equation turbulence model for aerodynamic flows," La Recherche Aérospatiale, , No. 1, 1994, pp. 5-21.

[12] Menter, F. R., "Two-equation eddy-viscosity turbulence models for engineering applications," AIAA Journal, Vol. 32, 1994, pp. 1598-1605. https://doi.org/10.2514/3.12149.

[13] Launder, B., Reece, G., and Rodi, W., "Progress in the development of a Reynolds-stress turbulence closure," Journal of Fluid Mechanics, Vol. 68, No. 3, 1975, pp. 537-566. https://doi.org/10.1017/s0022112075001814, URL/https://doi.org/10.1017/ s0022112075001814

[14] Speziale, C., Sarkar, S., and Gatski, T., "Modelling the pressure-strain correlation of turbulence: an invariant dynamical systems approach,” Journal of Fluid Mechanics, Vol. 227, 1991, pp. 245-272. https://doi.org/10.1017/s0022112091000101. URL https://doi.org/10.1017/s0022112091000101

[15] Cécora, R., Radespiel, R., Eisfeld, B., and Probst, A., "Differential Reynolds-Stress Modeling for Aeronautics," AIAA Journal, Vol. 53, No. 3, 2015, pp. 739-755. https://doi.org/10.2514/1.j053250 URL https://doi.org/10.2514/1.j053250

[16] Deck, S., "Recent improvements in the Zonal Detached Eddy Simulation (ZDES) formulation," Theoretical and Computational Fluid Dynamics, Vol. 26, 2012, pp. 523-550. https://doi.org/10.1007/s00162-011-0240-z

[17] Deck, S., Renard, N., Laraufie, R., and Weiss, P.-É., "Large-scale contribution to mean wall shear stress in high-Reynoldsnumber flat-plate boundary layers up to $R e_{\theta}=13650$," Journal of Fluid Mechanics, Vol. 743, 2014, pp. $202-248$. https://doi.org/10.1017/jfm.2013.629 
[18] Renard, N., and Deck, S., "Improvements in Zonal Detached Eddy Simulation for Wall Modeled Large Eddy Simulation," AIAA Journal, Vol. 53, 2015, pp. 3499-3504. https://doi.org/10.2514/1.j054143

[19] Deck, S., Weiss, P.-E., and Renard, N., "A rapid and low noise switch from RANS to WMLES on curvilinear grids with compressible flow solvers," Journal of Computational Physics, Vol. 363, 2018, pp. 231-255. https://doi.org/10.1016/j.jcp.2018. 02.028

[20] Vaquero, J., Renard, N., and Deck, S., "Effects of upstream perturbations on the solution of the laminar and fully turbulent boundary layer equations with pressure gradients," Physics of Fluids, Vol. 31, No. 12, 2019, p. 125103. https://doi.org/10.1063/ 1.5125496 URL https://doi.org/10.1063/1.5125496.

[21] Cuvier, C., Srinath, S., Stanislas, M., Foucaut, J. M., Laval, J. P., Kähler, C. J., Hain, R., Scharnowski, S., Schröder, A., Geisler, R., Agocs, J., Röse, A., Willert, C., Klinner, J., Amili, O., Atkinson, C., and Soria, J., "Extensive characterisation of a high Reynolds number decelerating boundary layer using advanced optical metrology," Journal of Turbulence, Vol. 18, 2017, pp. 929-972. https://doi.org/10.1080/14685248.2017.1342827

[22] Song, S., and Eaton, J. K., "Reynolds number effects on a turbulent boundary layer with separation, reattachment, and recovery," Experiments in Fluids, Vol. 36, 2004, pp. 246-258. https://doi.org/10.1007/s00348-003-0696-8

[23] Liou, M.-S., “A Sequel to AUSM: AUSM+," Journal of Computational Physics, Vol. 129, 1996, pp. 364-382. https: //doi.org/10.1006/jcph.1996.0256.

[24] Mary, I., and Sagaut, P., "Large Eddy Simulation of Flow Around an Airfoil Near Stall," AIAA Journal, Vol. 40, 2002 , pp. 1139-1145. https://doi.org/10.2514/2.1763

[25] Laraufie, R., Deck, S., and Sagaut, P., "A dynamic forcing method for unsteady turbulent inflow conditions," Journal of Computational Physics, Vol. 230, 2011, pp. 8647-8663. https://doi.org/10.1016/j.jcp.2011.08.012

[26] Head, M. R., and Bandyopadhyay, P., "New aspects of turbulent boundary-layer structure," Journal of Fluid Mechanics, Vol. 107, 1981, p. 297. https://doi.org/10.1017/s0022112081001791.

[27] Renard, N., and Deck, S., "On the scale-dependent turbulent convection velocity in a spatially developing flat plate turbulent boundary layer at Reynolds number $R e_{\theta}=13000$," Journal of Fluid Mechanics, Vol. 775, 2015, pp. 105-148. https://doi.org/10.1017/jfm.2015.290

[28] Radhakrishnan, S., Piomelli, U., Keating, A., and Lopes, A. S., "Reynolds-averaged and large-eddy simulations of turbulent non-equilibrium flows," Journal of Turbulence, Vol. 7, 2006, p. N63. https://doi.org/10.1080/14685240601047736.

[29] Spalart, P. R., Jou, W.-H., Strelets, M., and Allmaras, S. R., "Comments on the feasibility of LES for wings, and on a hybrid RANS/LES approach,” Proceedings of the First AFOSR International Conference on DNS/LES, Greyden Press, 1997, pp. $137-147$. 